\title{
El modelo de auditoria COSO III, para el sector salud en Villavicencio-Meta
}

\author{
The COSO III audit model, for the health sector in \\ Villavicencio-Meta
}

(1)

Martha L. Rey ${ }^{1}$

Johan E. Olmos Sabogal ${ }^{2}$

Mileny A. Rodríguez Molina ${ }^{3}$

\section{Artículo de investigación}

Recepción: 08-12-2020

Aceptación: 15-06-2021

El Conuco es una revista de acceso abierto revisada por pares. (C) $2018 \mathrm{El}$ autor (es). Este es un artículo de acceso abierto distribuido bajo los términos de la Licencia Internacional Creative Commons Attribution 4.0 (CC-BY 4.0), que permite el uso, distribución y reproducción sin restricciones en cualquier medio, siempre que se acredite el autor y la fuente originales.

Consulte http://creativecommons.org/licenses/by/4.0/. ऽOPEN ACCESS

1 Docente Ocasional Tiempo Completo; Magister en Fiscalidad Internacional, programa de Contaduría Pública, Facultad Ciencias Económicas, Universidad de los Llanos, correo institucional; mrey@unillanos.edu.co, Código ORCID: https://orcid.org/0000-0002-3985-7541

2 Estudiante de VI semestre, programa de Contaduría Pública, Facultad Ciencias Económicas, Universidad de los Llanos, correo institucional; johan.olmos@unillanos.edu.co, Código ORCID: https://orcid.org/0000-0002-5109-1593

3 Estudiante de VI semestre, programa de Contaduría Pública, Facultad Ciencias Económicas, Universidad de los Llanos, correo institucional; mileny.rodriguez@unillanos.edu.co, Código ORCID: https://orcid.org/0000-0003-1552-140X Este artículo hace parte del trabajo de aula del curso de Metodología de la Investigación diurnos del programa de Contaduría Pública.

Como citar este artículo / To reference this articcle:

Mileny A. (2020). El modelo de auditoria COSO III, O: (investigación, economía y sociedad), 3(1), pp. 1-12.

DOI: https://doi.org/10.22579/2619-614X.768

\section{Resumen}

La investigación busca profundizar en el conocimiento de la contabilidad y control desde una aproximación para conocer la concepción del sistema de control interno empleado en las Pymes. Es imprescindible reconocer la importancia del control interno, que para el caso de esta investigación se desarrolla con los componentes del modelo COSO III, en el contexto de las Pymes del sector salud.

La metodología empleada en la investigación, se desarrolla desde una perspectiva cuantitativa orientada hacia un enfoque descriptivo; la información se obtiene y se analiza mediante listas de chequeo y encuetas. El propósito de este estudio es determinar la efectividad del control interno implementado en las Pymes del sector salud, desde una aproximación teórica teniendo como marco de referencia el modelo de control interno propuesto por COSO III.

Palabras clave: Control, auditoria interna, Pyme, contabilidad

Código JEL: H2, M41, M42

\section{Abstract}

The research seeks to deepen the knowledge of accounting and control from an approach to understand the conception of the internal control system used in Pymes. It is essential to recognize the importance of internal control, which in the case of this research is developed with the components 
of the COSO III model, in the context of the Pymes in the health sector.

The methodology used in the research is developed from a quantitative perspective oriented towards a descriptive approach; the information is obtained and analyzed through checklists and surveys. The purpose of this study is to determine the effectiveness of the internal control implemented in the Pymes of the health sector, from a theoretical approach having as a reference framework the internal control model proposed by COSO III.

Keywords: control, internal audit, SME, accounting

\section{Introducción}

El control interno como objeto de estudio, es visto como herramienta indispensable para la gestión organizacional de las entidades que provee mecanismos para prevenir y/o evitar los diferentes riesgos a los que se enfrentan las Pymes en su funcionamiento. Proporcionando un interés para la prevención, acondicionándose a un buen crecimiento y el cumplimiento de sus objetivos, de manera en que sus resultados deben ser óptimos para generar nuevas propuestas, estrategias y pautas para la entidad.

Esta investigación parte desde una aproximación teórica, con el propósito de describir la efectividad del control interno implementado en las Pymes del sector salud, desde una revisión a la producción intelectual, teniendo como marco de referencia el modelo de control interno propuesto por COSO III.

La metodología empleada en la investigación, que se desarrollada desde una perspectiva cuantitativa orientada hacia un enfoque descriptivo mediante listas de chequeo y encuestas como instru- mentos para la recolección de datos y análisis de la información.

Este artículo está estructurado de la siguiente manera: Descripción y justificación del problema Aproximación Teórica y Conceptual, Marco Legal y Normativo, Marco contextual, Horizonte Metodológico y reflexión.

\section{Descripción y justificación del problema}

En el desarrollo de la formación adquirida en la universidad como contador público, surge el interés por la investigación acerca del control interno a partir del trabajo de aula realizado en los cursos de metodología de la investigación y control interno. Se observa la competencia de adentrarse en el conocimiento orientado hacia un contexto particular como son las Pyme del sector salud. El interés del equipo de trabajo nace a partir de la preocupación por la implementación de un sistema de control interno efectivo para este tipo de empresas.

Otros proyectos de investigación desarrollados sobre este objeto de estudio son: "Control Interno en Colombia: Un diagnóstico desde lo teórico" (López y Guevara, 2016, p. 243). En donde exploran las particularidades que deben tenerse en cuenta al abordar este objeto de investigación y utilizan una metodología de análisis documental entre los modelos de control interno que se aplican en Colombia y los modelos aplicados en países con los mejores indicadores de transparencia en el mundo. La corrupción que sufre la sociedad colombiana es un fenómeno político y económico que influye indirectamente a todos los sectores de la economía. Otro estudio realizado es denominado como: "Acercamiento al control interno en las Pymes colombiana" (Díaz, 2015, p.1). Devela la importancia del control interno y 
el modelo COSO como marco de referencia para la evaluación de los sistemas de control interno; este proyecto resalta la relevancia del control interno como un conjunto de procedimientos para que las entidades alcancen el éxito en sus operaciones. Otro estudio realizado es el ensayo argumentativo "El modelo COSO como herramienta de control interno en las Pymes de Colombia" (Gómez, 2018, p.1). Propone este modelo como una herramienta eficaz que logra reducir los riesgos y evitar el mal uso de recursos, ocasionado por algunas deficiencias y debilidades en el manejo operativo, administrativo y financiero; denotando el uso inadecuado de la informalidad que impide el éxito de una Pyme.

De conformidad con lo anterior, la falta de herramientas que faciliten el buen funcionamiento de un sistema de control interno es predominante a todo tipo de sociedad y entre estas a entidades del sector salud, que emplean diferentes modelos de control interno contable para el seguimiento y evaluación de sus actividades económicas, dado a las múltiples inconformidades que surgen a la hora de tomar decisiones para la gestión empresarial; según Beltrán (1999), se ha comprobado que quienes hacen pleno uso de esta importante técnica, como en hospitales que son entidades públicas ha obtenido mejor gestión administrativa por su eficiencia, efectividad y economía, y está plenamente respaldado por las metas, elementos y características del sistema.

En este sentido, la investigación contiene información acerca de qué tan indudable es acoger un sistema de control interno en el área salud, abstrayendo información relevante de cómo, por qué y para qué es necesario implementar un modelo de control interno en una Pyme.

Esta investigación surge de los conocimientos obtenidos en el curso de control interno Con- taduría Pública, curso metodología de la investigación. Para llevar a cabo la conceptualización y una posibilidad de visualizar más el concepto de control interno en el sector salud. Se percibe acerca del problema, estudios que muestran el funcionamiento del sistema de control interno en las Pyme, donde difícilmente muestran la efectividad de un modelo de control interno general en el sector salud, permitiendo acoger planes de organización y eficiencia operativa. Posteriormente el problema tendrá consecuencias indirectas en las entidades la efectividad que puede ofrecer el modelo COSO III como sistema de control interno en este sector. Catalogadas por el Estado colombiano como Pymes, a razón que el control interno es considerado un elemento fundamental para el funcionamiento óptimo de las entidades, como afirma Henao (2017):

se logra convertir en el complemento ideal de las operaciones cotidianas de una organización, sin importar si son de tipo contable o financiero, esto sustentado en que el control interno se acopla perfectamente a todos los ciclos, operaciones o procesos que se realicen en una compañía, independientemente de su carácter" (p. 13)

Esta definición se incorpora al marco teórico y referencial para construir los argumentos inherentes al sistema de control interno adecuado.

Las empresas al no llevar un control interno adecuado son más propensas a padecer problemas o fallas en los lineamientos administrativos establecidos, que conllevan a una solución inmediata pero no efectiva como es la duplicidad de funciones o el mal uso del capital de trabajo, entre otros. Es imprescindible determinar la repercusión de los elementos que constituyen el control interno y las características principales de los modelos que emplean algunas entidades del sector 
para el logro de metas trazadas en los objetivos empresariales.

Esta investigación aporta una revisión documental de estudios relacionados con este fenómeno de estudio, realizados en los últimos cinco años demostrando la relevancia de utilizar un control interno eficiente para el mejoramiento de los ciclos transaccionales en los que actúa el control interno, sirviendo de cimiento para esta investigación y como punto de partida de otra investigación que se desee llevar a cabo. La definición de efectividad de un modelo de control interno, se hace desde una aproximación teórica que emerge como resultado en el transcurso de la investigación.

Para cumplir con los objetivos de una auditoría interna, el objeto a estudiar es una pieza clave en la revisión de los procedimientos que se deben realizar para evitar los posibles riesgos y fraudes; además de comprobar que se cumplan los criterios establecidos previamente por las Normas Internacionales de Auditoría.

De acuerdo con el apartado anterior, se formula la siguiente pregunta de investigación: ¿Cuál es la efectividad del modelo Coso III como sistema de control interno en las Pymes del sector salud En Villavicencio?

\section{Aproximación Teórica y Conceptual}

La teoría general de control indica los conceptos, objetivos, evoluciones, funciones básicas y fundamentales del control; denominada como una agrupación de técnicas, procedimientos y acciones que velan por un equilibrio y desarrollo de una empresa; necesario para garantizar la regulación del sistema operacional del plan de negocios, comparando los logros obtenidos con las metas propuestas por la empresa; a su vez "a partir de la aplicación de la misma las personas reconocen y evidencian las desviaciones que se presentan en el desarrollo de un proceso, programa o proyecto"(Hurtado y Álvarez, 2016, p. 7). Con relación a lo anterior se determina y se generan nuevos parámetros para conseguir un balance o equilibrio de los intereses de la empresa, identificando cuales son las posibles acciones correctivas, al realizar una medición y evaluación del desempeño de la empresa; de modo que el control citado en Hurtado y Alvares (2016) se toma como herramienta, con el propósito de comprobar y asegurar los objetivos, o metas de una entidad, llegando a un acuerdo para poder alcanzar dichos propósitos. También se encarga de tener proteger los bienes materiales mediante procedimientos que "preserven los recursos físicos de la empresa asegurando que los agentes reciban no más que aquello a lo que ellos tienen derecho del fondo de recursos de la empresa" (Sunder, 1997, p. 169); de manera que, se debe tener en cuenta los parámetros y normas inherentes al control, para salvaguardar los activos de las entidades.

En las teorías de derecho desarrolladas por Hans Kelsen, indica que el normativismo, es impulsado para suscitar la jerarquía de las normas inmersas en el sistema jurídico del estado. Hans afirma que toda norma es válida únicamente de manera condicional. En el desarrollo de la filosofía de la ciencia, el profesor emérito Richard Mattessich propone la metodología CoNAT (Teoría normativo condicional), como "instrumento para alcanzar los fines propuestos por el sistema contable, lo que exige una jerarquía de objetivos, donde juega papel fundamental los juicios de valor" (Mejía, 2005, p. 158). Se necesita el uso de un modelo metodológico que se adapte a los requerimientos para el logro de sus objetivos.

Con relación al párrafo anterior la auditoría interna pertenece a los elementos que forma el 
control, no obstante es la inspección que se da a la gestión de una empresa, "a través de una permanente evaluación de los riesgos gestionados por la organización, cuya finalidad es el cumplimiento de los objetivos establecidos" (Montes, et al , 2017, p. 80). En vista que se puede acceder para realizar un examen sistemático, en el que se desarrollan juicios fundamentados en actividades que se realicen en la empresa. Autores como Almeida y Vásquez (2013, como se citó en Cevallos, et al, 2018) mencionan que:

La auditoría interna está encargada de monitorear y supervisar las actividades de la compañía para alcanzar los objetivos de la entidad, y realizan una investigación en la que la auditoría interna la utilizan como herramienta para la prevención y detección de fraudes empresariales. (p.16)

Se proporciona información basada en principios que son requeridos para dar las conclusiones en los informes; es importante reconocer como parte fundamental los informes de auditoría para la toma de decisiones en la organización.

La auditoría se caracteriza por depender de varios principios; estos principios ayudan a hacer de la auditoría una herramienta eficaz y confiable "en apoyo de las políticas y controles de gestión, proporcionando información sobre la cual una organización puede actuar para mejorar su desempeño” (Sánchez-Toledo y Asociados, 2008, párr. 1).

Los principios generales que direccionan el actuar y el alcance de la auditoría se extraen de los pronunciamiento del IAASB que por sus siglas en ingles es conocido como el Consejo de Normas Internacionales de Auditoría y Aseguramiento y se hallan entre la NIA (Norma Internacional de Auditoria) 200-299 en donde se exponen las responsabilidades del auditor independiente quien debe ejercer su juicio profesional de acuerdo a un conjunto de normas y principios para poder proporcionar conclusiones pertinentes.
Los modelos de auditoria se fundamentan en normas y principios que permitan delimitar el trayecto de un sistema de control en una organización. El encargado de la auditoría en el ejercicio de su profesión, ejercerá su juicio profesional y emitirá el tipo de opinión a que dé a lugar; argumentada en los hallazgos encontrados en el modelo de auditoria empelado. El auditor debe conocer, entender y analizar los procedimientos de control interno de la entidad. Se infiere que los controles para evitar y/o mitigar riesgos de error, fraude o de incorrección material, son evaluados por parte de la auditoria para comprobar si estos se están cumpliendo a cabalidad. El control interno se constituye un elemento fundamental e indispensable para las tareas desarrolladas en el desarrollo de la auditoria.

Se han publicado diferentes modelos de control interno y entre los más conocidos son: El MECI (Sector público en Colombia), el COSO (USA), el COCO (Canadá), el Cadbury (Reino Unido), King (Sudáfrica), el Vienot (Francia) y MICIL (adaptación del COSO para Latinoamérica). Estos modelos contemporáneos han sido desarrollados con el fin de contribuir al éxito las organizaciones. Según Rivas (2011), el modelo COSO, COCO y MICIL son los más empleados en el continente americano.

El marco integrado de control interno propuesto por COSO, (Committee of Sponsoring Organizations), contiene las principales directivas para la implementación, gestión y control de un sistema de control efectivo. Después de la primera versión del coso, surgen grandes cambios en la industria y en la tecnología que llevo a realizar ajustes en los modelos. En mayo del año 2013 está lista la actualización del COSO III que "consta de cinco categorías o componentes que la administración diseña y aplica para proporcionar la seguridad razonable de que sus objetivos 
de control se llevarán a cabo adecuadamente." (Quinaluisa, et al, 2018, p. 73). Esta actualización propone desarrollar el marco original, utilizando 17 principios y 79 puntos de enfoque con el objetivo de ampliar los conceptos y el alcance del control interno.

La estructura de los componentes del marco integrado COSO III, se dispone para diseñar y evaluar de manera objetiva la efectividad del sistema de control interno en una entidad. Los componentes contenidos en el Marco integrado COSO III son ambiente de control, evaluación de riesgos, actividad de control, información y comunicación, y monitoreo.

\section{Marco Legal y Normativo}

La normatividad vinculada a esta investigación está precedida por la Constitución Política de Colombia en el artículo 209 y 269 que dictamina la obligatoriedad de manejar un sistema de control interno. Para el sector público se radicó la ley 87 de 1993 donde materializa y determina las normas específicas para llevar el control interno. Las entidades del sector privado optan por este sistema mediante los criterios de la Ley 1314 de 2009 y el Decreto Único reglamentario 2420 de 2015 en donde se encuentran los requisitos para el tipo de empresa que deban aplicar normas de auditoría. El tipo de empresa se clasificó de acuerdo a la Ley 905 de 2004, modificada a la Ley 590 de 2000 para la clasificación del tamaño de las empresas.

\section{Marco contextual}

Con relación a lo anterior, en el siguiente apartado se describe lo que se conoce del problema actualmente.

Las Pymes del sector salud se han catalogado entidades que están en una línea constante en implementación de nuevas normas y acuerdos, donde se necesita de un sistema para mantener un control en su organización, a causa de los cambios propiciados por el sistema de salud en el país. "Los distritos y municipios cuentan con sus propias direcciones locales de salud, son responsables de hacer el diagnóstico de la situación de salud" (Guerrero, et al., 2011, p. 152); acatando las directrices del Ministerio de Salud, para el desarrollo y el análisis funcional del servicio de salud en Colombia.

En Villavicencio departamento del Meta, las entidades pertenecientes al servicio de salud, en el transcurso de los últimos años han presentado una serie de dificultades económicas, en las cuales de forma alguna han podido continuar ante las quejas hacia los diferentes niveles de prestación de la salud, de acuerdo con

La administración de las entidades de salud tanto pública como privada en Colombia ha sido diagnosticada suficientemente, identificando los principales problemas y planteando sus soluciones. De estas últimas destaca, desde el punto de vista teórico y práctico, un elemento de carácter constitucional, originado en la empresa privada, al que se asignó el nombre de "Sistema de Control Interno y de Gestión”. (Beltrán, 1999, p. 230)

Además, las entidades sector salud "diseñen técnicamente, implementen y se le esté realizando una evaluación permanente al Sistema de Control Interno y sus elementos, para garantizar el adecuado desempeño y el cumplimiento de la razón de ser, o sea que se cumpla la misión institucional" (Beltrán, p. 231). De manera cabe la posibilidad de asegurar un buen desarrollo organizacional con mayor eficiencia, eficacia y transparencia. Estas estrategias encaminadas a la formación de un ambiente estable en el servicio, 
digno de prestar a los ciudadanos del municipio, que pasan por diferentes necesidades y requieren del servicio de la salud.

En consecuente de lo anterior el departamento del Meta cuenta con 29 municipios, entre ellos Villavicencio, es la capital del departamento y principal centro urbano de la región, siendo el municipio con mayor posicionamiento de la ciudad más grande de los llanos orientales, como fuente comercial y de provisión para la región en cuanto a nivel económico y cultural de los llanos.

La investigación teórica se lleva a cabo en Villavicencio en el sector salud, que cuenta con un entramado empresarial de 20 entidades conformadas por clínicas, hospitales, centros de salud, EPS e IPS. En este contexto, se permite representar las condiciones que tiene el sector servicio salud en cuanto al control interno en las entidades públicas y privadas. Según la ley 87 de 1993, se establecen normas para el ejercicio del control interno en las entidades y organismos del estado. Aunque esta ley tiene como finalidad adoptarse en las entidades del estado; las entidades privadas han reconocido la importancia de adoptar un sistema de control interno para el logro de sus objetivos.

\section{Horizonte Metodológico}

Para el desarrollo de la investigación, se propone un tipo de investigación cuantitativa que "Utiliza la recolección de datos para probar hipótesis con base en la medición numérica y el análisis estadístico, con el fin establecer pautas de comportamiento y probar teorías" (Dalle, et al, 2005, p. 4). Sirve para una enumeración estadística de los datos obtenidos además de que establece la exactitud para el comportamiento de la muestra obtenida. En este sentido, para la investigación la información obtenida debe estar organizada y clasificada por datos de manera estadística; lo cual se requiere para conocer los hechos, estruc- turas, procesos que se manejan y esto a través de la medición de algunos elementos que hay en las entidades del sector servicio salud; para determinar el desarrollo, pautas y problemas centrales de las entidades.

Se toma en cuenta para el proceso investigativo, que "permite transformar rasgos de las unidades en variables de medición, y asignar un valor o categoría a cada una de estas unidades construidas" (Hernández, et al, 2014, p. 158). Para un apoyo, puesto que se tendrá claro a cuál información de las Pymes se puede acceder, para llegar a un informe; sustentado en encuestas y listas de chequeo, teniendo en cuenta la parte lógica y razonable

El enfoque de esta investigación es descriptivo, porque miden y evalúan diversos aspectos del fenómeno a investigar. "En un estudio descriptivo se selecciona una serie de cuestiones y se mide cada una de ellas independientemente, para así -y valga la redundancia- describir lo que se investiga” (Hernández, et al, 1997, p. 71)

Lo descriptivo intenta recopilar información cuantificable para ser utilizada en el análisis y clasificación de la información obtenida de las herramientas aplicadas en la muestra y población, midiendo de manera independiente cada concepto $\mathrm{y}$ variables que puedan incurrir en el proceso de investigación para la contribución estadística al objeto de estudio. Esto sirve para la recolección de datos e información sobre el control interno de las Pymes del sector salud, para realizar un análisis con relación al sistema propuesto por COSO III y presentar la efectividad que puede tener este modelo de control interno en las Pymes.

La población de esta constituida por 20 entidades del sector servicio de salud, ubicadas en la ciudad de Villavicencio departamento del Meta. Se analizarán y clasificaran las Pymes que conforman el entramado empresarial cumplen con los 
requisitos, para ser tomada como muestra en la propuesta de investigación.

En esta investigación, las Pymes que se tomarán como muestra, estas son las que reúnen los criterios establecidos para la aplicación de Normas internacionales de auditoría y que implementen características del modelo de control interno cosO III.

Para los propósitos de esta investigación de conformidad a la perspectiva cuantitativa y descriptiva se proponen las listas de chequeo y encuestas para recabar y analizar la información obtenida.

La lista de chequeo es pertinente para tener una estructura organizada en la recolección de información, para el análisis descriptivo que se emplea, es decir

"Se realiza una lista de acciones a realizar, elementos que conseguir, etc. Una vez que se vayan alcanzando los objetivos de la lista, se irán marcando. Proporcionando así una vista general y muy rápida del estado de las tareas. Como consecuencia, nos encontramos una mejora tanto en la productividad como en la eficiencia y optimización de los numerosos procesos de la empresa” (" ¿Cuál es la importancia de... ., 2018, párr. 2)

La lista de chequeo contiene información detallada y clara sobre los requisitos para clasificar las entidades del sector salud para reconocer si es catalogada como Pyme. Los requerimientos que deben cumplir estas Pymes, están estipulados de acuerdo a la Ley 590 de 2000 modificada por la Ley 905 de 2004, Ley 87 de 1993, y el Decreto único reglamentario 2420 de 2015 actualizado y con lo establecido en el artículo 209 del Código de comercio para revisores fiscales, Normas de Auditoria Generalmente Aceptadas. La lista de chequeo contendrá preguntas con respuestas cerradas, para el aporte de datos específicos, así mismo el análisis que se obtendrá, será elemental, sistemático y base para la propuesta de investigación. Se aplicará a algunos empleados de las entidades, coordinadores, gerente, contador y auditor interno de las Pymes del sector salud.

La encuesta se emplea con el fin de medir a las entidades aspectos estructurales; según Quispe y Sánchez (2011), la encuesta es una técnica de investigación que se efectúa mediante la elaboración de cuestionarios y entrevistas de manera verbal o escrita, su propósito es obtener información mediante el acopio de datos, mediante un análisis e interpretación, permitiendo tener una idea de la realidad para formar hipótesis.

Se realizarán varias encuestas semi-estructuradas verbales, para ir observando y analizando el manejo que han venido haciendo las Pymes del sector salud en relación al control interno. Se aplicará a gerentes, contador público y auditor interno de las empresas que apliquen del sector.

\section{Discusión}

En Colombia las Pymes del sector salud están obligadas a cumplir las normas de control interno para garantizar un buen sistema en calidad de salud; con el objetivo de mantener un equilibrio económico, mejorando los resultados de crecimiento, subsistencia y desarrollo. Es imprescindible que un sistema de control interno se adapte a nuevas circunstancias como riesgos y cambios que se puedan presentar en la organización.

El modelo de auditoria Coso III, es utilizado para mejorar el sistema de gestión de riesgos en las organizaciones; es necesario que una entidad mantenga claro sus objetivos, partiendo de esto se desarrolla una evaluación en dirección de su cumplimientos; Según Alarcón y Torres (2017), 
con el apoyo de planes estratégicos y planes de acción, se pueden lograr las metas establecidas desde las perspectivas de equidad, ética, eficiencia, efectividad para podrá tomar decisiones correctas y oportunas para mejorar la gestión de la organización, reduciendo así el impacto negativo.

Con la adopción de las Normas Internacionales de Auditoría (NIA) emitidas por la Federación Internacional de Contadores (IFAC), se obtiene un lenguaje común y avanzado en la evaluación del control interno al momento de realizar una auditoria efectiva. Es importante desarrollar in- vestigaciones que aborden las concepciones, principios y procesos de la información que cumplan con los requerimientos del nuevo lenguaje universal de las Normas Internacionales de información financiera y aseguramiento de la información en donde se encuentras las NIAS.

El modelo de auditoría es una herramienta efectiva para las Pymes de cualquier sector de la economía porque sus criterios de enfoque y evaluación contenidos en los componentes alcanzan a controlar todas las actividades y relaciones que lleva la entidad. 


\section{Referencias}

Alarcón, F. y Torres, M. (2017). Evaluación de control interno y gestión del riesgo aplicando el informe coso i, ii, iii; en los procesos administrativos y financieros de las entidades públicas. Revista publicando, 2 (11), 33-48. https:/ / revistapublicando.org/revista/index.php/crv/ article/view/544/pdf_368

Beltrán, l. (1999). Sistema de Control Interno en Entidades de Salud. Revista de salud pública, 1 (3). 230-234. http:/ / bdigital. unal.edu.co/22364/1/18964-61880-1PB.pdf

Blog Calidad y Excelencia. (21 agosto, 2018). ¿Cuál es la importancia de los checklist en una empresa? https://www.isotools. org/2018/08/21/importancia-dechecklist-en-una-empresa/

Cevallos, D., Moreno, C. J., y Chávez, Á. (2018), La auditoría interna como herramienta efectiva para la prevención de fraudes en las empresas familiares. Universidad y Sociedad, 10(5), 15-20. http:// scielo.sld.cu/pdf/rus/v10n5/22183620-rus-10-05-15.pdf

Dalle, P., Boniolo, P., Sautu, R. y Elbert, R. (2005). Manual de metodología. Construcción del marco teórico, formulación de los objetivos y elección de la metodología. CLACSO, Consejo Latinoamericano de Ciencias Sociales.http://bibliotecavirtual. clacso.org.ar/clacso/formacion-virtual/20100719035021/sautu.pdf

Decreto 0302 de 2015. (2015, 20 de febrero. Congreso de la República. Diario oficial
No 49.431. https://www.javeriana.edu. co/personales/hbermude/leycontable/contadores/2015-decreto-302.pdf

Decreto único reglamentario 2420 de 2015. (2015,14 de diciembre). Congreso de la República. Diario oficial No 49.726. https://www.cancilleria.gov.co/sites/ default/files/Normograma/docs/decreto_2420_2015.htm\#: :text=Por $\% 20$ medio $\% 20$ del $\% 20$ cual $\% 20$ se, y $\% 20$ se $\% 20$ dictan $\% 20$ otras $\% 20$ disposiciones.

Diaz, J. (2015), Acercamiento al control interno en las pymes colombiana. (Trabajo de grado. Universidad de La Salle). Archivo digital. https://ciencia.lasalle.edu.co/contaduria_publica/95/

Gómez, S. (2018). El modelo COSO como herramienta de control interno en las pymes Colombia. (Opción de grado, Universidad Militar Nueva Granada). Archivo digital. https:/ / repository.unimilitar.edu.co/ handle/10654/20780

Guerrero, R., Gallego, AI., Becerril-Montekio, V y Vásquez, J. (2011). Sistema de salud de Colombia. Salud Pública Mex. 53(2), 144-155. https://scielosp.org/pdf/ spm/2011.v53suppl2/s144-s155/es

Henao, Y. (2018). Importancia del control interno como berramienta en la detección y prevención de riesgos empresariales. (Trabajo de grado, Universidad del Valle, Cali, Colombia). Archivo digital https:// bibliotecadigital.univalle.edu.co/bitstream/handle/10893/11020/0567378. pdf? sequence $=1$ \&is Allowed $=y$ 


\section{Referencias}

Hernández, R., Collado, C. Y Baptista M. (2014). Metodología de la investigación 6ta edición. Interamericana editores, s.a. de c.v.. http://observatorio.epacartagena. gov.co/wp-content/uploads/2017/08/ metodologia-de-la-investigacion-sextaedicion.compressed.pdf

Hernandez, R., Fernández, C. y Baptista, P. (1997). Metodología de la Investigación. Hill Interamericana de Mexico, S.A. de C.V. https:/ /www.uv.mx/personal/cbustamante/files/2011/06/Metodologiade-la-Investigaci $\% \mathrm{C} 3 \% 83 \% \mathrm{C} 2 \% \mathrm{~B} 3 \mathrm{n}_{\text {_ }}$ Sampieri.pdf

Hurtado, M. y Álvarez, N. (2016). Teoría general del control tecnología contable y tributaria facultad de ciencias contables. Universidad Remington. http:/ /imagenes.uniremington. edu.co/moodle/M $\%$ C3\%B3dulos $\% 20$ de $\% 20$ aprendizaje/teoria $\% 20$ general $\% 20$ del $\% 20$ control $/$ Teoria $\% 20$ general $\% 20 \mathrm{del} \% 20$ control $\% 202016$.pdf

Ley 100 de 1993. (1993,23 de diciembre). Congreso de la República. Diario oficial No 41.148. http://www.secretariasenado.gov.co/senado/basedoc/ ley_0100_1993.html\#: :text=La\%20 Seguridad $\% 20$ Social $\% 20$ Integral $\% 20$ es, $1 \mathrm{a} \% 20$ cobertura $\% 20$ integral $\% 20$ de $\% 201$ as

Ley 1314 de 2009. (2009,13 de julio). Congreso de la República. Diario oficial No 47.409. http://www.secretariasenado.gov.co/senado/basedoc/ ley_1314_2009.html
Ley 87 de 1993. (1993,29 de noviembre). Congreso de la República. Diario oficial No 41.120. http://www.secretariasenado.gov.co/senado/basedoc/ ley_0087_1993.html

Ley 905 de 2004. (2004, 2 de agosto). Congreso de la República. Diario oficial No 45.628. http://www.secretariasenado.gov.co/senado/basedoc/ ley_0905_2004.html

López, O. y Guevara, J. (2016). Control Interno en Colombia: Un diagnóstico desde lo teórico. En-Contexto, 4 (5), 243-268. https:/ / ojs.tdea.edu.co/index.php/encontexto/article/view/377

Mejía, E. (2005). Introducción al pensamiento contable de Richard Mattessich. Revista Internacional Legis de Contabilidad Y Auditoría, (24),135-174. https:/ / xperta.legis. co/visor/temp_rcontador_161a1019be23-4e39-88d0-0c6aae708693

Montes, C., Porras, C., Muñoz, R. y Dextre, J. (2017). Auditoría Interna y Gestión Organizacional, revista proyecciones. (12), 69-95. https:// revistas.unlp.edu.ar/ proyecciones/article/view/6535/5580

Quinaluisa, N., Ponce, V., Muñoz, S., Ortega, X., y Pérez, J. (2018). El control interno y sus herramientas de aplicación entre COSO y COCO. Cofin Habana, 12(1). 268-283. http://scielo.sld.cu/scielo. php? script $=$ sci_arttext\&pid $=$ S207360612018000100018\&lng=es\&tlng=es. 


\section{Referencias}

Quispe, D, y Sánchez, G. (2011). Encuestas y entrevistas en investigación científica, Revista de Actualización Clínica. (10). 490494 http://www.revistasbolivianas.org. bo/pdf/raci/v10/v10_a09.pdf

Rivas, G. (2011). Modelos contemporáneos de control interno. Fundamentos teóricos. Observatorio Laboral Revista Venezolana,4(8).115-136. https://www.redalyc. org/pdf/2190/219022148007.pdf
Sánchez-Toledo \& Asociados. (2008). Los 7 principios de las auditorías de los sistemas de gestion. https://st-asociados. com/2018/10/los-7-principios-de-lasauditorias-de-los-sistemas-de-gestion/

Sunder, S. (2005). Teoría de la contabilidad y el control [N. García, Trad.;] [Trabajo original publicado en 1997]. http:/ /www.fce. unal.edu.co/publicaciones/images/pdf/ Teoria_de_la_contabilidad_2015.pdf 